\title{
LA VIE ET L'OEUVRE DE GASTON JÈZE'1
}

\author{
THE LIFE AND WORK OF GASTON JÈZE
}

Mariana Almeida Kato²

\begin{abstract}
Résumé
En 2 mars 1869 est né à Toulouse Gaston Jèze: un des plus importants juristes du droit public français, notamment du droit administratif et de finances publiques. En droit administratif, les théories qu'il a developpé dans le domaine du service public font de lui un des grands noms de l'École du Service Public fondé par Léon Duguit. En finances publiqeues, il est reconnu comme le " fondateur 》 français, ou en tous cas le « promoteur » de son enseignement autonome. Gaston Jèze a également développé une méthode d'étude du droit. Pour présenter les éléments de la vie et de l'œuvre de Gaston Jèze, le présent travail est divisé en deux sections. La première partie (section I) est dédiée à la biographie de Gaston Jèze. La deuxième partie (section II) est une analyse de sa méthode d'étude du droit et des ses principaux contributions pour le droit administratif et les finances publiques.
\end{abstract}

Mots-clés: Gaston Jese. Droit Administratif. Méthode d'étude du Droit.

\section{Abstract:}

In march 2nd 1869 Gaston Gesé was born. He was one of the most important jurists of French public law, notably of administrative and financial law. In administrative law the theories he developed on public domain of public services was source of great influence in the École du Service Public, founded by Léon Duguit. In financial law he is recognized as the " promotor » of the autonomy of the discipline. Gaston Jèze also developed a method of study in law. In presenting the elements of the life and work of Gaston Jesé, this paper divides in two sections. The fist (Section I) is dedicated to the biography of Gaston Jèze. The second part (Section II) is an analysis of the method of study of law and his main contributions to administrative and financial law.

Keywords: Gaston Jese. Administrative Law. Methods of Legal Teaching.

Sumário: 1 Introduction. Section I - La vie juridique et politique de Gaston Jèze. I.A - Gaston Jèze, le juriste théoricien du droit. I.B - Gaston Jèze, le personnage politique contesté. II - La contribution de Gaston Jeze au droit. II.A - La méthode developpé par Gaston Jèze. II.B - Les principales contributions au droit administratif et au droit des finances. 3. Conclusion. 4. Bibliographie.

1 Artigo submetido em 25/11/2015, pareceres de aprovação em 10/02/2016 e 18/02/2016, aprovação comunicada em 19/02/2016.

2 Advogada. Titular do Master em Direito Público pela Université Paris V Descarte, Paris (2015) e Mestranda em Direito Constitucional e Direitos Fundamentais na Université Paris I PanthéonSorbonne. E-mail: <mariana.kato@yahoo.com.br>. 
En 2 mars 1869 est né à Toulouse Gaston Jèze : un des plus importants juristes du droit public français, notamment du droit administratif et de finances publiques (WALINE, 1953, p. 886). En droit administratif, les théories qu'il a developpé dans le domaine du service public font de lui un des grands noms de l'École du Service Public fondé par Léon Duguit. En finances publiqeues, il est reconnu comme le « fondateur » français (COLLIARD, 1954, p. 11), ou en tous cas le «promoteur » de son enseignement autonome. Gaston Jèze a également développé une méthode d'étude du droit. Celle-ci est un élément marquant de ses ouvrages, en soulignant l'importance de la technique juridique pour l'étude du droit.

Son prestige est telle que sa contribution au droit dépasse même les frontières de la France (avec publication en Allemagne, par exemple) ${ }^{3}$ et de l'Europe (avec publications aux États-Unis et en Argentine) ${ }^{4}$. En réalité, on peut dire que son importance vient aussi d'une ouverture à l'international. II n'était pas seulement « exportateur » du droit français, mais « importateu » du droit étranger (BEAUD, 2014, p. 2). Sa bibliographie (pas exhaustive) qui a été publiée par la Revue de Droit Public en son hommage la prouve: six traductions, cinq ouvrages en langue étrangère et seize articles dans le domaine du droit international public (MURACCIOLE, 1953, p. 904-925).

Mais il était plus qu'un professeur, un théoricien ou un « savant » du droit comme il voulait être considerée (MILLET, 2007, p. 425). Gaston Jèze était aussi un personage politique important qui refusait certaines conventions (comme l'habitude des professeurs de droit de donner leurs cours en portant la robe) et s'élevait contre des régimes non républicains ou non démocratiques. Et donc, si d'un côté «Gaston Jèze théoricien » faisait l'unanimité, «Gaston Jèze praticien » créait la polémique.

Pour présenter les éléments de la vie et de l'œuvre de Gaston Jèze, le présent travail est divisé en deux sections. La première partie (section I) est dédiée à

3 «Das verwaltungsrecht des französische Republik», Bd. XXII. Das öffentliche Recht der Gegenswart. Tübbingen, Mohr, 1913, in-4, passim.

4 "The war finances of France. The war expenditure of France». New Haven, Yale, Unib. Press., 1927, gr, in-8으, passim et «Las finanzas publicas de la Republica argentina ». Buenos Aires, Ed. Le courrier de La Plata, 1924, gr., in-8o, passim. 
la biographie de Gaston Jèze. La deuxième partie (section II) est une analyse de sa méthode d'étude du droit et des ses principaux contributions pour le droit administratif et les finances publiques.

Mais avant tout, il convient de souligner la note que fait Rafael Bielsa au début son article en hommage à Gaston Jèze : «l'examen et l'analyse de l'oeuvre de Jèze méritent un livre et que cette brève note doit se limiter aux traits généraux » (BIELSA, 1953, p. 893).

\section{SECTION I - LA VIE JURIDIQUE ET POLITIQUE DE GASTON JEZE}

\section{I.A - GASTON JEZE, LE JURISTE THEORICIEN DU DROIT}

Gaston Jèze a fait toutes ses études à Toulouse. En 1892, à l'âge de 23 ans, il reçoit le titre de docteur après avoir présenté ses thèses en droit romain et droit civil (COLLIARD, 1954, p. 8-9).

Malgré sa formation « civiliste », son premier ouvrage important est consacré à l'étude du droit des finances publiques : Eléments de la science des finances et de la législation financière française, publié en 1896, avec son collègue Max Boucard (JÈZE ; BOUCARD, 1896, passim). Ce n'est pas le premier ouvrage sur les finances publiques qui a été publié, mais il « fait figure de nouveauté sinon de révolution » (COLLIARD, 1954, p. 8). À l'époque, « l'étude des finances publiques apparaît, en France au moins, comme un chapitre ou un tomme de l'économie politique » (COLLIARD, 1954, p. 16). L'ouvrage qu'ils ont publié est donc considerée comme le premier « véritable traité de finances publiques » ou même comme « une déclaration d'indépendance »(COLLIARD, 1954, p. 16). Puis, ce traité sera publié seulement par Gaston Jèze sous le titre: Cours élémentaire de science des finances et de législation financière française.

En 1897 et 1899 il a essuyé deux échecs au concours d'agrégation en droit public. Probablement, l'audace d'écrire un traité de finances publiques lui a coûté « la réussite à l'agrégation car le jeune docteur avait bousculé les usages et certains n'ont pas dû apprécier l'homme pressé qu'il paraissait être » (BEAUD, 2013, p. 2). Pourtant, le dernier échec lui a donné l'opportunité d'être chargé de cours à la 
Faculté de Droit d'Aix. Et en 1901 il réussi au concours de la Faculté de Droit de Lille et devient professeur. II dispense le cours de «droit administratif, jurisdiction et contentieux administratif »(MILLET, 2007, p. 425). En 1905 il devient «professeur titulaire » de droit administratif dans la même université.

Mas ce sont les années 1903-1904 qui peuvent être considérées comme étant les plus importants de sa carrière. Le premier événement marquant est la création avec Max Boucard de la Revue de science et de législation financière en 1903. Dans la présentation du programme de la Revue, ils écrivait : « Et cependant, la libre discussion scientifique des doctrines financières n'est-elle pas la condition indispensable de tout progrès serieux et durable ? » (JÈZE ; BOUCARD, 1903, p. 1). Leur programme était le débat ouvert sur la science des finances publiques, avec des textes de doctrine, des documents législatifs et aussi du droit comparé.

Le deuxième événement marquant est la publication de son premier commentaire d'arrêt dans L'année administrative qu'il a fondé avec Maurice Hauriou. L'importance de cette publication ne teint pas tant à son contenu, mais parce que il va dédier 40 ans de sa carrière à commenter des arrêts. (MILLET, 2007, p. 425). Et, comme explique Michel Waline, par cet exercice, «il a imposé, avec Maurice Hauriou «cette exigence du travail en réference perpétuelle à la jurisprudence » (WALINE, 1953, p. 879). Et c'est sourtout dans la Revue de droit public et de la science politique dont il prend la direction cette même année, qu'il publie ses commentaires d'arrêts.

Finalemment, c'est à cette date qu'il publie la premier édition de son livre Les principes généraux du droit administratif. Dans sa première édition, c'était l'indication d'une méthode pour le droit administratif français. La deuxième édition complète cet ouvrage en trois parties: la technique juridique, le service public et la fonction publique. Dans la troisième édition, chacune de ces parties devient un livre autonome ${ }^{5}$.

5 Tome 1 sur « la technique juridique du droit public français » en 1925, tomme 2 sur « la notion de service public et le statu des agents publics " en 1930 et tomme 3 sur «le fonctionemment des services publics, colaboration des particulier et contrats administratifs » en 1926 (SALON, 2005, p. 1 ; BEAUD, 2013, p. 6). 
Mais a cet instant, sa carrière ne fait que débuter. En 1908, il devient également directeur de la Revue pratique du contentieux et des impôts. Et, finalement, en 1909 il rejoind la faculté de droit de Paris où il reste jusqu'à 1937 « guère soutenu par ses collègues » (MILLET, 2007, p. 426). Le cours de droit public qu'il dispense dans cette université est par la suite publié en 7 volumes : retrait des actes juridiques (vol. 1), statut de la fonction publique (vol. 2 et 3), la théorie générale des contrats de l'administration (vol. 4, 5, 6 et 7). (SALON; SAVIGNAC, 2004). Même après sa retraite, il continue à travailler et il publie des commentaires d'arrêt jusqu'à 1948 - 5 ans avant sa mort (MILLET, 2007, p. 426).

Sa contribution pour le droit français est tellement importante que sa bibliographie ne comporte pas de liste exhaustive de ses publications dans la Revue de Droit Public (MURACCIOLE, 1953, p. 904/925). Cela résulte aussi du fait qu'il était aussi un «juriste résolument tourné vers l'international » (MILLET, 2007, p. 426). II a créé en 1927 l'Institut International de droit public pour réunir les grands noms de la discipline au niveau international « afin de contribuer au développement des libertés publiques par le droit dans les pays libres » (MILLET, 2007, p. 426). À la présentationde la Revue des finances, il écrivait avec Max Boucard « La législation comparée n'est-elle pas, en quelques sorte, un laboratoire de travaux pratiques pour les institutions sociales ?» (JÈZE ; BOUCARD, 1903, p. 3).

Gaston Jèze peut donc, être considéré comme un théoricien du droit dont le travail est remarcable et assez complet dans le domaine du droit public. Mais l'occasion de son retraît à la vie universitaire démontre qu'il n'était pas seulement un théoricien du droit, mais aussi un homme politique contesté à son époque.

\section{I.B - GASTON JEZE, LE PERSONNAGE POLITIQUE CONTESTE}

En plus des ses remarcables ouvrages et ses théories, Gaston Jèze est connu aussi par sa vie politique et ses opinions révolutionnaires ou contraires aux régimes autoritaires de son époque. Comme affirmé par Marc Milet : « La place et la posture tenue par Gaston Jèze au sein du corps des juristes ne sont pas étrangères 
à l'agitation: personalité austère et sévère, homme de gauche parmi un corps parisien largement acquis à la droit modérée [...] ». (v) (MILLET, 2007, p. 426). Un exemple de la personnalité marquante de Gaston Jèze est dans le fait qu'il ne faisait pas cours avec la robe, en opposition aux usages et à la tradition de l'époque. Jean Portemer a écrit sur la surprise des étudiants quand-ils voient un professeur sans robe : « mais la réalité est là, qui nous surprend. » (PORTEMER, 1991, p. 10).

Politiquement, c'est la première Guerre Mondiale qui a amené Gaston Jèze à s'engager pour la défense des valeurs républicaines. En 1914 il participe au « front du droit » qui réunissait les juristes françaises pour contester radicalement les doctrines allemandes qui légitimaient le conflit (MILLET, 2007, p. 426). A la fin de la Guerre, il devient conseiller du prince, « expert auprès des autorités publiques ». A partir de 1923, il remplit plusieurs missions pour le gouvernement en Argentine, Chili, Roumanie, Yougoslavie - qui ont contribué à ses publications en droit comparée. ${ }^{6} \|$ milite aussie dans des associations promouvant la Socité des Nations.

Qualifié à l'époque d'" homme de gauche, proche du milieu radical socialiste » (MILLET, 2007, p. 425), il se présent aussi sur une liste du «parti socialiste colonial » en Guadeloupe aux élections législatives de 1919. Mais il est battu et établit un an plus tard « une note très critique sur l'état économique et moral de la colonie dans laquelle il dénonce l'attitude des élites politiques et administratives. » (MILLET, 2007, p. 425).

En 1924 il participe à l'ouvrage « la politique républicaine » « qui fait office de manifeste du cartel des Gauches » (MILLET, 2007, p. 426). En 1926 il devient membre «du comité des experts qui conduit à infléchir la politique monétaire gouvernamentale et de la Banque de France»(MILLET, 2007, p. 426). Dans les annés 30, il est conseilleur du Négus de l'Ethiopie. A cette occasion, il a défendu la cause de l'Éthiope envahie par l'Italie fasciste de Moussolini et a dénoncé le régime autoritaire, en provoquant une véritable crise politique (MILLET, 2007, p. 425). C'est donc à l'occasion de la seconde Guerre Mondiale que Gaston Jèze devient l'un des premiers grands juristes français pour protester contre le régime nazi antisémite et raciste (MILLET, 2007, p. 426).

6 À titre d'exemple, on peut citer l'article : « La question des optants hangrois de Transylvanie devant la S.D.N. dans la Réforme agraire en Roumanie et les optants hangrois, Paris, 1928, p. 92 . 
En raison de ces opinions politiques et cette posture, il a été plusieurs fois attaqué par des étudiants royalistes et nationalistes qui l'empêchaient de donner son cours (MILLET, 2007, p. 426). De même, ses collègues à l'Université de Paris étaient toujours irrités par lui pour les mêmes raisons. Ainsi, M. Millet rapporte que « certains de ses propos tenus dans ses cours sur le régime napoléonien ou la diplomatie française avaient provoqué quelques remous » (MILLET, 2007, p. 426). Selon Rafael Bielsa (1953, p. 892), ce sont les raisons qui lui ont coûté sa retraite à l'université.

Mais ces élements de la personnalité et de la vie politique de Gaston Jèze ne sont pas indépendent de ses théories. On voit que "ses convictions et prises de position publiques apparaissent au final étroitement liées à ses travaux 》 (BIELSA, 1953, p. 892).

\section{II - LA CONTRIBUTION DE GASTON JEZE AU DROIT}

\section{II.A - LA METHODE DEVELOPPE PAR GASTON JEZE}

Comme Jöel Molinier affirme que " c'est avant tout par la méthode d'étude des phénomènes financier qu'il a formulée et utilisée que Gaston Jèze a fait oeuvre novatrice » (MOLINIER, 1991, p. 70). Dans le préface de la troisième édition de son livre des principes juridiques, il marque bien l'importance de cette méthode : «plus j'avance en âge, plus je suis convaincu que la seule chose qui importe pour l'étude du droit, c'est une bonne méthode. II n'y a qu'une seule bonne méthode : c'est la méthode d'observation des faits » (JÈZE, 2005, p. I, préface).

L'affirmation ci-dessus est révélatrice de sa technique juridique : l'étude des «faits » ou du « millieu ». Selon les mots de Gaston Jèze : «Le Droit vit ; il évolue sans cesser comme tout ce qui vi, sous l'influence du milieu. Observons sans cesser ce millieu : nous comprendrons mieux le Droit ; nous l'adapterons mieux aux besoins du moment » (JÈZE, 2005, p. V, préface). II faut que les juristes voient le droit comme un phénomène en mouvement constant qui dialogue avec la réalité où il s'impose. Comme Olivier Baud affirme : " le substrat du droit est un substrat social, 
donc vivant [...] II en résulte une osmose étroite entre le droit et les faits sociaux qui était déjà soulignée par Duguit et que Jèze reprend entièrement à son compte » (BEAUD, 2013, p. 6). Donc, selon Gaston Jèze, « [...] toute théorie qui ne cadre pas avec les faits porte en elle-même sa condamnation, son germe de mort. » (JÈZE, 2005, p. XI, préface).

Comme résultat de cette vision, « en rupture à la fois avec les approches praticienne et scolastique de la discipline, Gaston Jèze a milité pour que la science juridique porte sur l'analyse des causes et des conditions dans lesquelles se posent les problèmes juridiques » (MILLET, 2007, p. 426). En plus, cela résulte aussi de sa position contraire à l'histoire du droit (et notamment contre le droit romain), lorsque «Selon Jèze, on ne peut jamais expliquer rétrospectivement les anciennes mentalités ». Pour lui, le « droit romain est du droit mort » (BEAUD, 2013, p. 7). C'est pour cette raison que sa méthode est insérée dans le cadre d'un « positivisme sociologique » (MASLARSKI, 2009. p. 2), « qui voit dans le droit, l'ensemble des règles de conduite sociale estimées justes et socialement utiles par la majorité d'une population à une époque donné » (MILLET, 2007, p. 426).

Par conséquence, Gaston Jèze refuse une étude du droit centré seulement sur les textes juridiques - et précisément en raison de cette dimension dynamique du droit. Comme l'indique Michel Waline, «il ne faut donc pas confondre les études juridiques avec l'effort de mémoire, consistant à apprendre le détail de la législation et de la réglementation en vigueur à un instant de l'Histoire » (WALINE, 1953, p. 880). Pour Gaston Jèze, les textes législatifs et réglementaires sont des éléments « fragiles et instables. » (SALON ; SAVIGNAC, 2004). Ainsi, il affirme « combien de livres de Droit, écrits d'après la conception soi-disant «pratique » sont rapidement rendus inutiles à raison d'un changement de la législation et de la jurisprudence » (JÈZE, 2005, p. II, préface).

Cela explique sa militance en faveur d'un enseignement du droit fondé sur l'éducation scientifique e non sur la préparation professionnel. En se posant la question sur le rôle des facultés de droit sur leur mission de former des jurisconsultes ou prépares des avocats et des juges, il réponde sans hésitation : 
jurisprudence, le plus gros de ses connaissances s'évanouisse. C'est l'éponge passé sur l'ardoise. II n'y reste plus rien. C'est une instruction à refaire. (JÈZE, 2005, p. II, préface)

Mais Gaston Jèze ne rejette pas l'étude des ces textes (WALINE, 1953, p. 880). Il est aussi considéré comme un positiviste (BIELSA, 1953, p. 896). Mais pour lui l'étude du droit positif doit être accompagnée des «études économiques, sociologiques, politiques [qui] doivent être à la base des études juridiques (JÈZE, 2005, p. II, préface). Le danger est que, sans ces études, « on forme bien des légistes, mais non des jurisconsultes; on prépare des empiriques, mais non des savants. » (JÈZE, 2005, p. II, préface). Cette vision lui à amener à défendre ce qu'il a appelé de « synthèse critique ».

Comme affirme Olivier Beaud, pour Gaston Jèze le juriste ne doit pas se limiter à faire à travail descriptif des textes car «il n'est pas un compilateur, ni un collectionneur ni même un classificateur. » (BEAUD, 2013, p. 6). C'est en cela que le juriste de doctrine se distingue du praticien. A partir de d'un travail de systématisation, le « juriste doit « théoriser » le droit positif » (BEAUD, 2013, p. 6). Comme l'explique Olivier Beaud, les solutions juridiques des théoriciens « ne sont pas créées par le juriste de doctrine de sorte que son rôle est forcément limité par cet objet duquel il ne peut jamais s'émanciper.» (BEAUD, 2013, p. 6). Cette « théorisation » n'est plus que le résultat du dialogue entre le droit en vigueur et la pensé du juriste théoricien sur ce droit (a partir des élements du millieu social) (JÈZE, 2005, p. II, préface).

L'application de cette méthode est commune à tous les domaines que Gaston Jèze a étudié. Comme le souligne Maurice Duverger, « il ne néglige jamais l'examen de ce qu'il nomme "les points de vue politique, social, et économique »: au contraire, il les associe à toute analyse juridique, souvent de façon minutieuse et détaillé » (DUVERGER, 1954, p. 21-22). La seule différence est dans l'important d'un élément particulier dans les diverses disciplines (DUVERGER, 1954, p. 21-22). Donc, « Jèze juriste financier n'est pas différent de Jèze juriste tout court : le premier ne fait que transposer dans un domaine particulier, en les adaptant, les principes et les techniques définis par le second »(DUVERGER, 1954, p. 21). 
En droit des finances, selon Colliard, « l'étude ne doit pas se borner à isoler tel ou tel aspect des problèmes financiers », « ne pas apparaître comme une simple partie d'un examen de caractère purement économique »(COLLIARD, 1954, p. 12). Comme Gaston Jèze explique dans son Cours de science des finances et de législation financière française: «pour ma part, je crois qu'il faut étudier les phénomènes financiers en tenant compte de tous les facteurs, non seulement des facteurs financiers économiques, mais aussi des facteurs politiques, sociaux, juridiques. » (JÈZE, 1922, p. 8). Et c'est l'élément politique qui, pour l'auteur, doit recevoir une primauté, lorsque le budget est considéré comme un acte essentiellement politique (COLLIARD, 1954, p. 13; JĖZE, 1922, p. I, préface).

En droit administratif, le méthode n'est pas different. II fait une differenciation entre les points de vue juridique et politique du droit administratif pour défendre leur complementarité dans l'étude du droit (BEAUD, 2013, p. 8). Comme il affirme :

\begin{abstract}
II faut toujours rechercher le besoin social, politique, économique, auquel correpond telle institution ; les conditions économiques, sociales, politiques dans laquelles fonctionne tel ou tel service public ; le rendement pratique, au point de vue social, politique, économique, du service. Tout cela, c'est le point de vue politique. II faut aussi examiner les règles et les procédés juridiques au moyen desquels tel but est porsuivi et atteint ; par lesquels tel service public fonctionne. Tout cela, c'est le point de vue juridique. (JÈZE, 2005, p. 3)
\end{abstract}

C'est cette differenciation de points de vue qu'il va utiliser pour critiquer les théories dont il est en désaccord. Comme l'affirme Olivier Beaud : « il lui fait jouer un rôle discriminant qui en fait une arme polémique dirigée contre les thèses doctrinales avec lesquelles il est en désaccord » (BEAUD, 2013, p. 8) - comme le droit naturel (WALINE, 1953, p. 880-881).

Mais, malgré sa défense pour l'étude complémentaire de ces deux élements, après «il assimile progressivement le «technique » au «juridique » et expulse le politique du juridique »(BEAUD, 2013, p. 10). Donc, il est possible d'affirmer qu'il n'était pas complètement fidèle à sa méthode (BEAUD, 2013, p. 10). Ce que fait Oliver Beaud affirmer que « son œuvre juridique doit être considérée comme « une œuvre de l'entre-deux »(BEAUD, 2013, p. 10). 


\section{II.B - LES PRINCIPALES CONTRIBUTIONS AU DROIT ADMINISTRATIF ET AU DROIT DES FINANCES}

Le rapport de Gaston Jèze au droit n'est pas limité au droit administratif et aux finances publiques. Comme l'analyse Marcel Waline, il a consacré aussi d'importantes études au domaine du droit constitutionnel et international (WALINE, 1953, p. 887). Mais c'est plutôt dans ces deux domaines qu'il a consacré sa carrière. Et malgré la grande diversité qui peut être considérée comme presque exhaustive dans ces domaines (comme on peut le voir dans sa bibliographie), on centrera cette étude sur les éléments que demeurent les plus actuels aujourd'hui.

En droit administratif, comme Olivier Baud l'affirme, « il a écrit non seulement quantité d'articles de droit administratif (notamment sur le statut des fonctionnaires et la responsabilité de l'État) mais aussi un nombre encore plus considérable de notes de jurisprudence. » (BEAUD, 2013, p. 2). Mais il est plus connu par sa liasion avec l'École de Service Public de Léon Duguit qui fonde la théorie du droit public sur la notion de «service public » (BEAUD, 2013, p. 4).

Pour Gaston Jèze, le service public est vu «comme nouvelle notion fondamentale du droit public moderne » - «même que le champ d'activité de l'état s'étend, que le critère de définition et de distinction se révèle inopérant, entre acte de gestion et acte d'autorité » (MILLET, 2007, p. 426). Pour lui, "Le droit public et administratif est l'ensemble des règles relatives aux services publics, et, pour le fonctionemment régulier de ces services, il existe nécessairement des règles juridiques spéciales » (JĖZE, 2005, p. 1). En d'autres termes, «le droit public et le droit admministratif en particulier étaient, pour lui, « l'ensemble des règles relatives au service public » (SALON ; SAVIGNAC, 2004, p. 5). Ces règles sont des règles juridiques spéciales que ramènent à l'idée d'intérêt général devant laquelle l'interêt particulier doit s'incliner (JĖZE, 2004, p. 3). En bref, pour Gaston Jèze, le service public est la «pierre angulaire » du droit administratif (MILLET, 2007, p. 426).

En droit des finances publiques, il était précurseur sur quelques points de la science des finances - comme la conception du budget, du partage des 
compétances financières entre Gouvernement et Parlement, et sa critique à « l'esprit de fiscalité »(MOLINIER, 1991, p. 69). Et c'est plutôt dans ce domaine qu'on voie des réflexes de sa pensé politique.

Comme affirme Henry Lauffenburger: «Tout en l'élevant contre la conception communisante de l'impôt, Jèze accorde nettement sa préférance à l'impôt sur le revenu au taux progressif en tant que se rapprochant le plus de « l'idéal » de la justice fiscale » (LAUFENBURGER, 1954, p. 29). Dans sa théorie du budget, il fait un approche assez multidisciplinaire en reconnaissance « qu'inévitablement, par la redistribution qu'impliqe le budget, les gouvernements modifient la quote-part des diverses classes dans le revenu national et aussi dans la fortune nationale, qu'ils affesctent la structure sociale »(LAUFENBURGER, 1954, p. 26-27). Par contre et malgré ses convictions politiques, il reffusait une vision communiste des finances «car si elle triomphait, les finances ne seraient plus que politiques, les points de vue technique et économique seraient sacrifiés en violation du caaractère universaliste de la science »- ce qui peut être expliqué en raison de la technique qu'il applique à l'étude du droit (LAUFENBURGER, 1954, p. 26-27). Mais, comme Jöel Mollinier l'affirme :

Le contenu proprement juridique de l'oeuvre de Jèze ne peut guère de nos jours retenir l'attention, non pas tant parce que les règles de droit positif du temps de l'auteur, auxquelles ce dernier a été amené à fare une place, ont bien évidemment pour la plupart cessé de s'applique, mais parce que la réflexion juridique de Jèze ayant pout objet les finances publiques a souvent été encore plutôt depassés. (MOLINIER, 1991, p. 69)

C'est donc pour deux raisons que son importance est aussi vivante de nos jours. D'abord, en raison de sa volonte d'émancipation du droit des finances publiques et de sa contribution à l'ériger en science autonome. Le livre Eléments de la science des finances et de la législation financière française, écrit avant même que la science des finances soient considerée comme une science autonome en France, en somme avec la création de la Revue des finances publiques sont des contributions incommensurables pour la science qui est étudié aujourd'hui de façon autonome. En plus, il n'a pas cessé pendant toute sa vie de continuer à développer des études dans tous les domaines des finances publiques (MOLINIER, 1991, p. 69). 
En plus, son approche plurisdisciplaire de la discipline - et donc de sa «technique juridique » appliqué au droit des finances publiques - est assez remarcable et très audacieuse pour son époque. La façon dont le droit des finances publiques est abordé aujourd'hui avec un regard multidisciplinaire doit beaucoup à sa contribution (MOLINIER, 1991, p. 69).

L'importance de Gaston Jèze pour le droit public ne peut être méconnue. Selon l'affirmation de Michel Waline, « dans le domaine du droit public, aucun juriste français, si ce n'est outrefois Duguit, n'a atteint, et il s'en faut de beaucoup, la renommée de Gaston Jèze. » (WALINE, 1953, p. 879). C'est possible d'affirmer que cette renommée vient du passé qu'il a contruit : une vie dédiée à l'étude du droit, ses plusieurs publications et les discussions qu'il a provoqué.

Toutefois, ses ouvrages ne sont pas très utilisés aujourd'hui par rapport à ceux de Maurice Hauriou, par exemple (BEAUD, 2013, p. 2). Mais ça ne peut être vu comme marquant une absence de postérité. C'est une fausse conclusion. L'absence d'utilisation de certaines théories ne limite pas sa contribution au droit du temps où il a vécu. D'abord, car il a contribué pour la création d'une science autonome du droit des finances publiques, ce qui a des réflexes jusqu'à nos jours. En deuxième, en raison du fait qu'il était un juriste qui a tourné le droit au milleu social sans oublier la technique juridique - méthode aujourd'hui bien pacifique dans la doctrine, mais dans lequelles il etait un des principaux défenseurs. Comme c'est affirmé dans la préface de son livre «II ne peut y avoir de doute, Gaston Jèze est bien un auteur contemporain dont on a encore beaucoup à apprendre » (SALON ; SAVIGNAC, 2004, p. 8).

\section{BIBLIOGRAPHIE}

BEAUD, Olivier. «L'œuvre de Gaston Jèze signifie-t-elle un repli de la doctrine publiciste française sur la technique juridique ? ». Jus Politicum, Mutations du droit public, n. 11, décembre 2013. Disponible en : <http://juspoliticum.com/article/Loeuvre-de-Gaston-Jeze-signifie-t-elle-un-repli-de-la-doctrine-publiciste-francaise-surla-technique-juridique-825.html>. Acèss en : 15.01.2014. 
BERTHÉLEMY, Henry. Notice «Gaston JÈZE ». In : Nos maîtres de la faculté de droit de Paris. Paris : Librairie Générale de Droit et de Jurisprudence, 1932.

BIELSA, Rafael. «Le Professeur Gaston Jèze. Quelques réflexions que suggère son oeuvre ». Traduit par MURACCIOLE, Luc. Revue du droit public et de la science politique en France et à l'étranger, n. 2, p. 891/903, Avril/Juin 1953. Paris : Librarie Générale de Droit et de Jurisprudence.

COLLIARD, Claude-Albert. "Gaston Jèze, théoricien des finances publiques ». In : RIST, Charles; COLLIARD, Claude-Albert ; DUVERGER, Maurice. Hommage à Gaston Jèze. Paris : L.G.D.J., 1954, p. 8-18.

DUVERGER, Maurice. "Gaston Jèze, Juriste financier ». In: RIST, Charles ; COLLIARD, Claude-Albert; DUVERGER, Maurice. Hommage à Gaston Jèze. Paris : L.G.D.J., 1954. p. 19-23.

JĖZE, Gaston ; BOUCARD, Max. « Notre programme ». In : Revue de Science et de législation financières (directeurs: : JEZE, Gaston; BOUCARD, Max). Première année. Paris : V. GIARD \& E. BRIĖRE, 1903, p. 1/3.

JĖZE, Gaston. Cours de science des finances et de la législation financière française. $6^{\text {ème }}$ ed. Paris : Giard, 1922.

JÈZE, Gaston. Les principes généraux du droit administratif : la technique juridique du droit public français. Tome I. $3^{\text {ème }}$ édition. Paris : Dalloz, 2005.

JĖZE, Gaston. Les príncipes généraux du droit administratif. Tome II. Paris Dalloz, 2004.

LAUFENBURGER, Henry, «Gaston Jèze, économiste financier ». In : RIST, Charles; COLLIARD, Claude-Albert; DUVERGER, Maurice. Hommage à Gaston Jèze. Paris : L.G.D.J., 1954. p. 24-32.

MASLARSKI, David. "La conception de l'État de Gaston Jèze ». Jus Politicum, Autour de la notion de Constitution, n. 3, décembre 2009. Disponible en: $<$ http://juspoliticum.com/article/La-conception-de-I-Etat-de-Gaston-Jeze-147.html>.

Acèss en : 15.01.2014.

MILLET, Marc. Notice «Gaston Jèze ». In :Dictionnaire historique des juristes français : XIle - Xxe siècle (sous la direction de : ARABEYRE, Patrick ; HALPÉRIN, Jean-Louis ; KRYNEN, Jacques). Paris : PUF, 2007.

MOLINIER, Jöel. «L'apport de Gaston Jèze à la théorie des finances publiques ». Colloque Gaston Jèze. Revue d'histoire des facultés de droit et de la science juridique, n. 12, p. 55-70, 1991.

MURACCIOLE, Luc. «Biographie des oeuvres de Gaston Jèze ». In :Revue du droit public et de la science politique en France et à l'étranger. Avril-Juin 1953. № 2.

Paris : Librarie Générale de Droit et de Jurisprudence. p. 904/925.

PORTEMER, Jean. Avant-propos «Colloque Gaston Jèze ». In: Revue d'histoire des facultés de droit et de la science juridique. 1991. № 12. p. 9-13.

SALON, Serge ; SAVIGNAC, Jean-Charles. Préface. In : JÈZE, Gaston. Les príncipes généraux du droit administratif. Tome II. Paris Dalloz, 2004.

WALINE, Michel. «L'oeuvre de Gaston Jèze en Droit Public ». In : Revue du droit public et de la science politique en France et à l'étranger. Avril-Juin 1953, n. 2. Paris : Librarie Générale de Droit et de Jurisprudence. p. 879/890. 